\title{
Classification of functions with trivial solutions under t-equivalence
}

\author{
Yanqing Li ${ }^{\mathrm{a}, \mathrm{b}}$, Donghe Pei ${ }^{\mathrm{a}, *}$, Dejian Huang ${ }^{\mathrm{a}, \mathrm{b}}$, Ruimei Gao ${ }^{\mathrm{c}}$ \\ a School of Mathematics and Statistics, Northeast Normal University, Changchun, Jilin 130024, P. R. China. \\ ${ }^{b}$ School of ocean information engineering, Hainan Tropical Ocean University, Sanya, Hainan 572022, P. R. China. \\ ${ }^{c}$ Department of Science, Changchun University of Science and Technology, Changchun, Jilin 130022, P. R. China. \\ Communicated by K. Q. Lan
}

\begin{abstract}
We apply singularity theory to study bifurcation problems with trivial solutions. The approach is based on a new equivalence relation called t-equivalence which preserves the trivial solutions. We obtain a sufficient condition for recognizing such bifurcation problems to be $t$-equivalent and discuss the properties of the bifurcation problems with trivial solutions. Under the action of t-equivalent group, we classify all bifurcation problems with trivial solutions of codimension three or less. (C)2017 All rights reserved.
\end{abstract}

Keywords: Singularity, bifurcation, t-equivalence, classification.

2010 MSC: 58C25, 58K40.

\section{Introduction}

Bifurcation theory is the study of equations with multiple solutions. For a wide variety of equations, including many partial differential equations, problems concerning multiple solutions can be reduced to study how the solutions $x$ of a single scalar equation $g(x, \lambda)=0$ vary with the parameter $\lambda$. It is difficult to classify the qualitatively different equations because there are infinitely many equation types and there are equation types of arbitrarily high complexity. The singularity theory notion of codimension provides a rational approach to this problem. Golubistky and Schaeffer [6] introduced the idea of applying singularity theoretic methods to the study of bifurcation problems. Subsequently, some authors have studied classifications of bifurcation problems up to some codimensions. Most of low-codimension classifications has been performed. Such classifications include bifurcation problems in one state variable without symmetry up to codimension 7 (see [8]), those in one state variable with $\mathbb{Z}_{2}$ symmetry up to codimension 3 (see [7]), those in two state variables with $\mathbb{D}_{4}$ symmetry up to topological codimension 2 (see [5]) and with hidden symmetry (see [9]), and those in one state variable with two bifurcation parameters up to codimension 1 (see [11]). Apart from those, Gao and Li [3] discussed the classification of $\left(\mathbb{D}_{4}, S^{1}\right)$-equivariant bifurcation problems up to topological codimension 2.

\footnotetext{
${ }^{*}$ Corresponding author

Email addresses: liyq516@nenu.edu.cn (Yanqing Li), peidh340@nenu.edu.cn (Donghe Pei), hdj1107@qzu.edu.cn (Dejian Huang), gaorm135@nenu. edu.cn (Ruimei Gao)

doi:10.22436/jnsa.010.07.18
} 
The bifurcation theory may apply to the models from mathematical biology. In evolutionary theory, changes in the environment are often reflected by changes in the residents' ability to reproduce. Evolutionary game theory studies the evolution of phenotypic traits and was originated by Maynard-Smith and Price [12]. The adaptive dynamics approach for studying evolution of phenotypic traits has been explored by various authors such as Dieckmann and Law [2], Geritz et al. [4], Diekmann [1], and Waxman and Gavrilets [15]. Vutha and Golubitsky [13] applied singularity theory and adaptive dynamics theory to study ESS and CvSS singularities of strategy functions. Wang and Golubitsky [14] studied the fitness functions in adaptive dynamics under dimorphism equivalence and classified singularities up to topological codimension 2 . We present these here because these give the studying vitality by making connections with applications.

This paper applies the techniques from singularity theory to bifurcation problems with trivial solutions. The equivalence relation is called as t-equivalence which preserves the trivial solution. The equivalence was described in [7]. We give a sufficient condition to recognize bifurcation problems be equivalent. By analyzing the properties of germs, we give the classification theorem up to codimension 3 . All undefined terms and symbols could be seen in $[7,10]$. We suppose the function germs are smooth in this paper.

The structure of this paper is as follows. We present the basic notations and preliminaries in Section 2. In Section 3, we give a sufficient condition to judge t-equivalent. In Section 4, we discuss the properties of bifurcation problems and obtain the classification theorem of them.

\section{Basic concepts and preliminaries}

Let $\varepsilon_{x, \lambda}$ denote the space of germs of $C^{\infty}$ functions of $\left(\mathbb{R}^{2}, 0\right) \rightarrow \mathbb{R}$ with coordinates $x$ and $\lambda$. Let $\varepsilon_{\lambda}$ be the space of germs depending on one variable $\lambda$. A germ $g \in \varepsilon_{x, \lambda}$ satisfying $g(0,0)=g_{x}(0,0)=0$ is called a bifurcation problem at origin.

Definition 2.1. Let $g \in \varepsilon_{x, \lambda}$ be a bifurcation problem and $g(0, \lambda) \equiv 0$ for any $\lambda \in(\mathbb{R}, 0)$. Then we call $g$ a bifurcation problem with trivial solution.

Let $g$ be a germ in $\varepsilon_{x, \lambda}$ with $g(0, \lambda) \equiv 0$. Then there exists a smooth germ $f$ in $\varepsilon_{x, \lambda}$ such that

$$
g(x, \lambda)=x f(x, \lambda)
$$

Let $\varepsilon_{x, \lambda}\{x\}$ denote the subring of $\varepsilon_{x, \lambda}$ consisting of germs with trivial solutions, that is,

$$
\varepsilon_{x, \lambda}\{x\}=\left\{g \in \varepsilon_{x, \lambda} \mid g(x, \lambda)=x f(x, \lambda), f \in \varepsilon_{x, \lambda}\right\} .
$$

As a real vector space, $\varepsilon_{x, \lambda}\{x\}$ is a subspace of $\varepsilon_{x, \lambda}$. Let $I \subseteq \varepsilon_{x, \lambda}$. We denote the set $\{x f(x, \lambda) \mid$ for all $f \in I\}$ by $I\{x\}$.

Definition 2.2. Let $g, h \in \varepsilon_{x, \lambda}\{x\}$. Then we say $g$ and $h$ are t-equivalent, denoted $g \sim h$, if there exist a diffeomorphism $(X, \Lambda): \mathbb{R}^{2} \rightarrow \mathbb{R}^{2}$ and a function $S \in \varepsilon_{x, \lambda}$ such that the relation

$$
g(x, \lambda)=S(x, \lambda) h(X(x, \lambda), \Lambda(\lambda))
$$

holds near the origin. Here we still require that

$$
S(0,0)>0, X_{x}(0,0)>0, \Lambda_{\lambda}(0)>0, \Lambda(0)=0, X(0, \lambda) \equiv 0, \lambda \in(\mathbb{R}, 0) .
$$

The set of all $(S,(X, \Lambda))$ is a group, and denoted by $\Gamma$. In particular, $(S, \Phi)^{-1}=\left(\frac{1}{S}, \Phi^{-1}\right)$, where $\Phi=(X, \Lambda)$.

Let $(S,(X, \Lambda)) \in \Gamma, g \in \varepsilon_{x, \lambda}\{x\}$. Then the action of $\Gamma$ on $\varepsilon_{x, \lambda}\{x\}$ is defined by

$$
(S,(X, \Lambda)) g(x, \lambda)=S(x, \lambda) g(X(x, \lambda), \wedge(\lambda)) .
$$

The orbit of $g$ under the action of $\Gamma$ is $O_{g}=\left\{h \in \varepsilon_{x, \lambda}\{x\} \mid h \sim g\right\}$. 
Definition 2.3. Let $g \in \varepsilon_{x, \lambda}\{x\}$ be a bifurcation problem. The orbit tangent space of $g$ is defined by

$$
\widetilde{\mathrm{T}}(\mathrm{g})=\left\{\mathrm{ag}+\mathrm{bxg} g_{x}+\mathrm{c} \lambda g_{\lambda} \mid \mathrm{a}, \mathrm{b} \in \varepsilon_{x, \lambda}, c \in \varepsilon_{\lambda}\right\} .
$$

If $g(x, \lambda)=x f(x, \lambda)$, then $\widetilde{T}(g)$ can be represented by

$$
\widetilde{T}(g)=<g, x g_{x}>_{\varepsilon_{x, \lambda}}+\varepsilon_{\lambda}\left\{\lambda g_{\lambda}\right\}=\left[<f, x f_{x}>_{\varepsilon_{x, \lambda}}+\varepsilon_{\lambda}\left\{\lambda f_{\lambda}\right\}\right]\{x\} .
$$

Definition 2.4. Let $g \in \varepsilon_{x, \lambda}\{x\}$ satisfying $g=g_{x}=0$ at $(0,0)$.

(1) Let $W$ be a subspace of some vector space $X$. If there exists a subspace $V$ with finite dimension such that $X=W+V$, then we call $W$ has finite codimension in $X$.

(2) If $\widetilde{T}(g)$ has finite codimension in $\varepsilon_{x, \lambda}\{x\}$, then we call $g$ has finite codimension in $\varepsilon_{x, \lambda}\{x\}$.

(3) Let $r \in \mathbb{N}$. If there exists a vector subspace $V$ of $X$ with dimension $r$, such that $X=W \oplus V$, then $r$ is called the codimension of $W$ in $X$, denoted by $\operatorname{codim}_{X} W=r$ or $\operatorname{codim} W=r$. If $\operatorname{codim}_{\varepsilon_{x, \lambda}\{x\}} \tilde{T}(g)=r$, then we call $g$ has codimension $r$, denoted by codim $g=r$.

Remark 2.5. Let $W$ be a subspace of $\varepsilon_{x, \lambda}\{x\}$ with finite codimension. Set $W^{\prime}=\left\{h \in \varepsilon_{x, \lambda} \mid x h(x, \lambda) \in W\right\}$. Then it is easy to see that $W=W^{\prime}\{x\}$, where $W^{\prime}$ is a subspace of $\varepsilon_{x, \lambda}$ with finite codimension.

\section{The orbit tangent space}

Lemma 3.1. Let $\mathrm{g}, \mathrm{p} \in \mathcal{E}_{\mathrm{x}, \lambda}\{\mathrm{x}\}$ be bifurcation problems. If

$$
\tilde{\mathrm{T}}(\mathrm{g}+\mathrm{tp})=\tilde{\mathrm{T}}(\mathrm{g})
$$

holds for $\mathrm{t}$ near 0 , then there exist $\alpha, \beta, \gamma \in \varepsilon_{x, \lambda, t}$ such that

$$
p=\alpha G+\beta \chi G_{x}+\gamma \lambda G_{\lambda},
$$

where $G(x, \lambda, t)=g(x, \lambda)+\operatorname{tp}(x, \lambda)$.

Proof. Let $\mathrm{t}_{0} \in(\mathbb{R}, 0)$ satisfy $\tilde{\mathrm{T}}\left(\mathrm{g}+\mathrm{t}_{0} \mathrm{p}\right)=\tilde{\mathrm{T}}(\mathrm{g})$. Notice that

$$
\tilde{T}\left(g+t_{0} p\right)=<g+t_{0} p, x g_{x}+t_{0} x p_{x}>_{\varepsilon_{x, \lambda}}+\varepsilon_{\lambda}\left\{\lambda g_{\lambda}+t_{0} \lambda p_{\lambda}\right\}, \quad \tilde{T}(g)=<g, x g_{x}>_{\varepsilon_{x, \lambda}}+\varepsilon_{\lambda}\left\{\lambda g_{\lambda}\right\} .
$$

Since $g+t_{0} p, x g_{x}+t_{0} x p_{x}, \lambda g_{\lambda}+t_{0} \lambda p_{\lambda} \in \tilde{T}\left(g+t_{0} p\right)$, we have $g+t_{0} p, x g_{x}+t_{0} x p_{x}, \lambda g_{\lambda}+t_{0} \lambda p_{\lambda} \in \tilde{T}(g)$, and thus there exist germs $a_{i}, b_{i} \in \varepsilon_{x, \lambda}$ and $c_{i} \in \varepsilon_{\lambda}, i=1,2,3$ such that

$$
\begin{aligned}
g+t_{0} p & =a_{1} g+b_{1} x g_{x}+c_{1} \lambda g_{\lambda}, \\
x g_{x}+t_{0} x p_{x} & =a_{2} g+b_{2} x g_{x}+c_{2} \lambda g_{\lambda}, \\
\lambda g_{\lambda}+t_{0} \lambda p_{\lambda} & =a_{3} g+b_{3} x g_{x}+c_{3} \lambda g_{\lambda} .
\end{aligned}
$$

By (3.3), we have

$$
\left(\begin{array}{c}
p \\
x p_{x} \\
\lambda p_{\lambda}
\end{array}\right)=Q\left(\begin{array}{c}
g \\
x g_{x} \\
\lambda g_{\lambda}
\end{array}\right),
$$

where $Q$ is a $3 \times 3$ matrix whose entries are smooth germs in $\varepsilon_{x, \lambda}$. Now for any germ $h$, we introduce the notation $\mathrm{V}(\mathrm{h})$ for the column vector

$$
V(h)=\left(\begin{array}{c}
h \\
x h_{x} \\
\lambda h_{\lambda}
\end{array}\right) .
$$


Using this notation, we rewrite (3.4) in the form

$$
\mathrm{V}(\mathrm{p})=\mathrm{QV}(\mathrm{g})
$$

Recalling that $G=g+t p$, we get

$$
V(G)=V(g)+t V(p) .
$$

By (3.6) and (3.5), we have

$$
(\mathrm{I}+\mathrm{tQ}) \mathrm{V}(\mathrm{p})=\mathrm{QV}(\mathrm{G})
$$

Observe that (3.7) is a system of equations with smooth dependence on $t$. Since I is invertible, it follows that for sufficiently small $t, I+t Q$ is an invertible $3 \times 3$ matrix. Thus $(I+t Q)^{-1}$ is a $3 \times 3$ matrix whose entries are smooth germs in $\varepsilon_{x, \lambda, t}$. In particular, these germs are smooth in $t$. The invertibility of $I+t Q$ and (3.7) imply

$$
\mathrm{V}(\mathrm{p})=(\mathrm{I}+\mathrm{tQ})^{-1} \mathrm{QV}(\mathrm{G})
$$

Equating the first components on each side of (3.8) yields the equality

$$
p=\alpha G+\beta x G_{x}+\gamma \lambda G_{\lambda}
$$

where $\alpha, \beta$ and $\gamma \in \varepsilon_{x, \lambda, t}$.

Lemma 3.2. Let $\mathrm{g}, \mathrm{p} \in \varepsilon_{\mathrm{x}, \lambda}\{\mathrm{x}\}$ be bifurcation problems. If

$$
\tilde{\mathrm{T}}(\mathrm{g}+\mathrm{tp})=\tilde{\mathrm{T}}(\mathrm{g})
$$

holds for $\mathrm{t}$ near 0 , then $\mathrm{g}+\mathrm{tp} \sim \mathrm{g}$ for all sufficiently near 0 .

Proof. Let $\mathrm{G}(x, \lambda, \mathrm{t})=\mathrm{g}(x, \lambda)+\mathrm{tp}(x, \lambda), \mathrm{t} \in(\mathbb{R}, 0)$. By Lemma 3.1, there exist $\alpha, \beta, \gamma \in \varepsilon_{x, \lambda, t}$ such that (3.2) is true. The non-autonomous system

$$
\left(\begin{array}{l}
\dot{u} \\
\dot{v}
\end{array}\right)=\left(\begin{array}{l}
-u \beta(u, v, t) \\
-v \gamma(u, v, t)
\end{array}\right),\left\{\begin{array}{l}
u(0)=x \\
v(0)=\lambda
\end{array}\right.
$$

and the equation

$$
\left\{\begin{array}{l}
\dot{S}=-S \alpha(X(x, \lambda, t)), \Lambda(\lambda, t), t) \\
S(0)=1
\end{array}\right.
$$

decide the diffeomorphism family $(X(x, \lambda, t), \Lambda(\lambda, t))$ in $\left(\mathbb{R}^{2}, 0\right)$ for $t \in(\mathbb{R}, 0)$ and $S \in \varepsilon_{x, \lambda, t}$. Besides, the solutions of the above system satisfy $X(0, \lambda, t) \equiv 0, \Lambda(0, t) \equiv 0, t, \lambda \in(\mathbb{R}, 0)$. Thus we get a family of triples $(S(x, \lambda, t),(X(x, \lambda, t), \Lambda(\lambda, t))) \in \Gamma, t \in(\mathbb{R}, 0)$ which satisfy

$$
S(x, \lambda, t) G(X(x, \lambda, t), \wedge(\lambda, t), t)=g(x, \lambda) .
$$

In fact,

$$
\begin{aligned}
& \frac{d}{d t}(S(x, \lambda, t) G(X(x, \lambda, t), \Lambda(\lambda, t), t)) \\
& \quad=S_{t}(x, \lambda, t) G(X, \Lambda, t)+S(x, \lambda, t)\left[G_{X}(X, \Lambda, t) X_{t}+G_{\lambda}(X, \Lambda, t) \wedge t+G_{t}(X, \Lambda, t)\right] \\
& \quad=-S \alpha(X, \Lambda, t) G(X, \Lambda, t)-S\left[G_{X}(X, \Lambda, t) X \beta(X, \Lambda, t)+G_{\lambda}(X, \Lambda, t) \wedge \gamma(X, \Lambda, t)-p(X, \Lambda)\right]=0 .
\end{aligned}
$$

Thus

$$
S(x, \lambda, t) G(X(x, \lambda, t), \wedge(\lambda, t), t)=S(x, \lambda, 0) G(X(x, \lambda, 0), \wedge(\lambda, 0), 0)=g(x, \lambda) .
$$

This completes the proof. 
Theorem 3.3. Let $\mathrm{g}, \mathrm{p} \in \mathcal{E}_{\mathrm{x}, \lambda}\{x\}$ be bifurcation problems. If (3.1) holds for all $\mathrm{t} \in[0,1]$, then $\mathrm{g}+\mathrm{t} \mathrm{p} \sim \mathrm{g}$ for all $\mathrm{t} \in[0,1]$.

Proof. Let $\mathrm{t}_{0} \in[0,1]$ and $\mathrm{h}=\mathrm{g}+\mathrm{t}_{0} \mathrm{p}$. Then

$$
\tilde{\mathrm{T}}(\mathrm{h})=\tilde{\mathrm{T}}(\mathrm{g})
$$

and

$$
\tilde{\mathrm{T}}(\mathrm{h}+\mathrm{rp})=\tilde{\mathrm{T}}\left(\mathrm{g}+\left(\mathrm{t}_{0}+\mathrm{r}\right) \mathrm{p}\right)=\tilde{\mathrm{T}}(\mathrm{g})
$$

for all $r$ sufficiently near 0 . By (3.9) and (3.10), we have

$$
\tilde{\mathrm{T}}(\mathrm{h}+\mathrm{rp})=\tilde{\mathrm{T}}(\mathrm{h}) .
$$

By Lemma 3.2, we get $h+r p \sim h$. Then $g+\left(t_{0}+r\right) p \sim g+t_{0} p$, that is,

$$
g+t p \sim g+t_{0} p
$$

for all $t$ near $t_{0}$. Thus the equivalence classes of $t^{\prime} s$ are open and it follows from either the compactness or connectedness of $[0,1]$ that there is exactly one equivalence class. Hence $g+t p \sim g$ for all $t \in[0,1]$.

\section{Main results}

Lemma 4.1. Let $g \in \varepsilon_{x, \lambda}\{x\}$ be singular at $(0,0)$ with finite codimension. Then there exists an integer $l \geqslant 1$ such that $\mathrm{g}$ can be written as $\mathrm{g}=\mathrm{x}(\mathrm{h}+\mathrm{k})$, where $\mathrm{h} \in \varepsilon_{\mathrm{x}}$ is l-regular for $\mathrm{x}$ and $\mathrm{k} \in\left\langle\lambda>_{\varepsilon_{\mathrm{x}, \lambda} \lambda}\right.$.

Proof. Let $g(x, \lambda)=x f(x, \lambda)$, where $f \in \varepsilon_{x, \lambda}$. Then

$$
g_{x}(x, \lambda)=f(x, \lambda)+x f_{x}(x, \lambda) .
$$

Observe that $g$ is singular at origin. Then $f \in M_{x, \lambda}$, where $M_{x, \lambda}$ is the maximal ideal in $\varepsilon_{x, \lambda}$.

Set $k(x, \lambda)=f(x, \lambda)-f(x, 0), h(x)=f(x, 0)$. Then $k \in\left\langle\lambda>_{\varepsilon_{x, \lambda}}\right.$ and $h \in M_{x}$, where $M_{x}$ is a maximal ideal in $\varepsilon_{x}$. Now we prove that there exists an integer $l \geqslant 1$ such that $h \in \varepsilon_{x, \lambda}$ is l-regular for $x$. Let

$$
W=W^{\prime}\{x\}=\tilde{T}(g) .
$$

Since $g$ has finite codimension,

$$
W^{\prime}=<f, x f_{x}>_{\varepsilon_{x, \lambda}}+\varepsilon_{\lambda}\left\{\lambda f_{\lambda}\right\}
$$

is a subspace of $\varepsilon_{x, \lambda}$ with finite codimension. Let $\Phi: \varepsilon_{x, \lambda} \rightarrow \varepsilon_{x}$ be an algebra homomorphism induced by inclusion map $i:(\mathbb{R}, 0) \rightarrow(\mathbb{R} \times \mathbb{R},(0,0)), i(x)=(x, 0)$. Then $\Phi$ is a surjection homomorphism. Thus $\Phi$ maps a finite subspace of $\varepsilon_{x, \lambda}$ to a finite subspace of $\varepsilon_{x}$. Especially, it is not difficult to show that

$$
\Phi\left(W^{\prime}\right)=<\Phi(f), \Phi\left(x f_{x}\right)>_{\varepsilon_{x}}
$$

is a subspace with finite codimension and also an ideal of $\varepsilon_{x}$. Note that $\Phi(f)=h, \Phi\left(x f_{x}\right)=x h_{x}$. Then there exists an integer $m \geqslant 1$ such that $M_{x}^{m} \subset<h, x h_{x}>_{\varepsilon_{x}}$. So there exist $a, b \in \varepsilon_{x}$ such that

$$
x^{m}=a h+x b h_{x} .
$$

Consider the series jet nets $j^{0} h(0), j^{1} h(0), j^{2} h(0), \ldots$. Note that $h \in M_{x}$. Then $j^{0} h(0)=0$. There must exist an integer $l \geqslant 0$ such that

$$
j^{0} h(0)=j^{1} h(0)=\cdots=j^{l-1} h(0)=0, j^{l} h(0) \neq 0 .
$$

Otherwise, there will be a contradiction by making m-jet net on two sides of (4.1). By Taylor theorem, $h \in \varepsilon_{x}$ is l-regular for $x$. 
Lemma 4.2. Let $g \in \varepsilon_{x, \lambda}\{x\}$ be a bifurcation problem and let $g(x, \lambda)=x f(x, \lambda)$, where $f \in \varepsilon_{x, \lambda}$. Then the following conditions hold.

(1) $\tilde{\mathrm{T}}(\mathrm{g}) \subset<x^{\mathrm{l}}, \lambda>\{x\}$ and codim $g \geqslant l$.

(2) If $l=3$ and $f_{\lambda}(0,0)=0$, then $\tilde{\mathrm{T}}(\mathrm{g}) \subset<x^{3}, x \lambda, \lambda^{2}>\{x\}$ and codim $g \geqslant 4$.

(3) If $\mathrm{l}=2$ and $\mathrm{f}_{\lambda}(0,0)=0$, we have

(i) if $f_{x \lambda}(0,0)=0$, then $\tilde{\mathrm{T}}(\mathrm{g}) \subset<x^{2}, \lambda^{2}>\{x\}$ and codim $g \geqslant 4$;

(ii) if $\mathrm{f}_{\lambda \lambda}(0,0)=0$, then $\tilde{\mathrm{T}}(\mathrm{g}) \subset<x^{2}, x \lambda, \lambda^{3}>\{x\}$ and codim $\mathrm{g} \geqslant 4$;

(iii) if $\mathrm{f}_{x \lambda}(0,0) \mathrm{f}_{\lambda \lambda}(0,0) \neq 0$, then $\tilde{\mathrm{T}}(\mathrm{g}) \subset<x^{2}, x \lambda, \lambda^{2}>\{x\}$ and codim $\mathrm{g} \geqslant 4$.

(4) If $l=1$ and $f_{\lambda}(0,0)=f_{\lambda \lambda}(0,0)=f_{\lambda \lambda \lambda}(0,0)=0$, then $\tilde{T}(g) \subset<x, \lambda^{4}>\{x\}$ and codim $g \geqslant 4$. Here we denote $<\mathrm{h}, \mathrm{k}>$ as $<\mathrm{h}, \mathrm{k}>_{\varepsilon_{\mathrm{x}, \lambda}}$.

Proof. By Lemma 4.1, let $f(x, \lambda)=x^{l} a(x)+\lambda q(x, \lambda)$, where $l \geqslant 1, a \in \varepsilon_{x}, q \in \varepsilon_{x, \lambda}$ and $a(0) \neq 0$.

(1) The codimension of $\left\langle x^{l}, \lambda>\{x\}\right.$ is l. Since $\tilde{T}(g) \subset<x^{l}, \lambda>\{x\}$, we have codim $\tilde{T}(g) \geqslant l$, it follows that codim $g \geqslant l$.

It is an easy task to verify the statements on codimension from the statement on containments. For example, consider (3). If $l=2$ and $f_{\lambda}(0,0)=0$, then there exist $q_{1}, q_{2} \in \varepsilon_{x, \lambda}$ such that $q=x q_{1}+\lambda q_{2}$. Thus

$$
f(x, \lambda)=x^{2} a(x)+x \lambda q_{1}+\lambda^{2} q_{2}
$$

(i) If $f_{x \lambda}(0,0)=0$, then there exist $q_{3}, q_{4} \in \varepsilon_{x, \lambda}$ such that $q_{1}=x q_{3}+\lambda q_{4}$ and so

$$
f=x^{2} a(x)+x^{2} \lambda q_{3}+x \lambda^{2} q_{4}+\lambda^{2} q_{2}
$$

and $f, x f_{x}, \lambda f_{\lambda} \in<x^{2}, \lambda^{2}>$. Thus

$$
\tilde{\mathrm{T}}(\mathrm{g}) \subset<x^{2}, \lambda^{2}>\{x\} .
$$

Since $\operatorname{codim}\left(<x^{2}, \lambda^{2}>\{x\}\right)=4$, we have codim $\tilde{T}(g) \geqslant 4$ and so codim $g \geqslant 4$.

(ii) If $\mathrm{f}_{\lambda \lambda}(0,0)=0$, then there exist $\mathrm{q}_{3}, \mathrm{q}_{4} \in \varepsilon_{x, \lambda}$ such that $\mathrm{q}_{2}=x \mathrm{q}_{3}+\lambda \mathrm{q}_{4}$ and so

$$
f=x^{2} a(x)+x \lambda q_{1}+x \lambda^{2} q_{3}+\lambda^{3} q_{4}
$$

and $f, x f_{x}, \lambda f_{\lambda} \in<x^{2}, x \lambda, \lambda^{3}>$. Thus

$$
\tilde{\mathrm{T}}(\mathrm{g}) \subset<x^{2}, x \lambda, \lambda^{3}>\{x\} .
$$

Since $\operatorname{codim}\left(<x^{2}, x \lambda, \lambda^{3}>\{x\}\right)=4$, codim $g \geqslant 4$.

(iii) If $\mathrm{f}_{x \lambda}(0,0) \mathrm{f}_{\lambda \lambda}(0,0) \neq 0$, then $\mathrm{q}_{1}(0,0) \cdot \mathrm{q}_{2}(0,0) \neq 0$. By (4.2), we have

$$
\begin{aligned}
f(x, \lambda) & =x^{2} a(x)+x \lambda q_{1}+\lambda^{2} q_{2}, \\
x f_{x}(x, \lambda) & =x^{2}\left[2 a(x)+x a_{x}(x)\right]+x \lambda\left[q_{1}+x q_{1_{x}}\right]+x \lambda^{2} q_{2_{x}} \\
\lambda f_{\lambda}(x, \lambda) & =x \lambda\left[q_{1}+\lambda q_{1_{\lambda}}\right]+\lambda^{2}\left[2 q_{2}+\lambda q_{2_{\lambda}}\right] .
\end{aligned}
$$

Hence $\tilde{T}(g) \subset<x^{2}, x \lambda, \lambda^{2}>\{x\}$. Since codim $\left(<x^{2}, x \lambda, \lambda^{2}>\{x\}\right)=3$, we have codim $g \geqslant 3$.

Next, we need to prove codim $g \neq 3$. It suffices to verify that $\left\langle x^{2}, x \lambda, \lambda^{2}>\{x\}\right.$ is not contained in $\tilde{T}(g)$.

Suppose that $<x^{2}, x \lambda, \lambda^{2}>\{x\} \subset \tilde{T}(g)$. Then

$$
<x^{2}, x \lambda, \lambda^{2}>c<f, x f_{x}>+\varepsilon_{\lambda}\left\{\lambda f_{\lambda}\right\} .
$$


Hence there exist $b_{i j} \in \varepsilon_{x, \lambda}, c_{i} \in \varepsilon_{\lambda}$, where $i=1,2,3$ and $j=1,2$, such that

$$
x^{2}=b_{11} f+b_{12} x f_{x}+c_{1} \lambda f_{\lambda}, \quad x \lambda=b_{21} f+b_{22} x f_{x}+c_{2} \lambda f_{\lambda}, \quad \lambda^{2}=b_{31} f+b_{32} x f_{x}+c_{3} \lambda f_{\lambda} .
$$

We calculate the 2-jet of equalities in (4.3) at the origin:

$$
\begin{aligned}
\frac{1}{2} \chi^{2} f_{x \chi}(0,0)+\chi \lambda f_{x, \lambda}(0,0)+\frac{1}{2} \lambda^{2} f_{\lambda \lambda}(0,0) & =a(0) x^{2}+q_{1}(0,0) x \lambda+q_{2}(0,0) \lambda^{2}, \\
\chi^{2} f_{x \chi}(0,0)+\chi \lambda f_{x, \lambda}(0,0) & =2 a(0) x^{2}+q_{1}(0,0) x \lambda \\
x \lambda f_{x, \lambda}(0,0)+\lambda^{2} f_{\lambda \lambda}(0,0) & =q_{1}(0,0) x \lambda+2 q_{2}(0,0) \lambda^{2} .
\end{aligned}
$$

Calculating the 2-jet of the first equality in (4.4) at the origin, we get

$$
\begin{aligned}
x^{2}= & b_{11}(0,0)\left[\frac{1}{2} \chi^{2} f_{x \chi}(0,0)+x \lambda f_{x, \lambda}(0,0)+\frac{1}{2} \lambda^{2} f_{\lambda \lambda}(0,0)\right] \\
& +b_{12}(0,0)\left[x^{2} f_{x x}(0,0)+\chi \lambda f_{x, \lambda}(0,0)\right]+c_{1}(0)\left[x \lambda f_{x, \lambda}(0,0)+\lambda^{2} f_{\lambda \lambda}(0,0)\right] .
\end{aligned}
$$

By (4.5) and (4.6), we have

$$
\begin{aligned}
x^{2}= & b_{11}(0,0)\left[a(0) x^{2}+q_{1}(0,0) x \lambda+q_{2}(0,0) \lambda^{2}\right] \\
& +b_{12}(0,0)\left[2 a(0) x^{2}+q_{1}(0,0) x \lambda\right]+c_{1}(0)\left[q_{1}(0,0) x \lambda+2 q_{2}(0,0) \lambda^{2}\right] .
\end{aligned}
$$

Then

$$
x^{2}=a(0)\left[b_{11}(0,0)+2 b_{12}(0,0)\right] x^{2}+q_{1}(0,0)\left[b_{11}(0,0)+b_{12}(0,0)+c_{1}(0)\right] x \lambda+q_{2}(0,0)\left[b_{11}(0,0)+2 c_{1}(0)\right] \lambda^{2} .
$$

Notice that $a(0) q_{1}(0,0) q_{2}(0,0) \neq 0$. Then

$$
\begin{aligned}
b_{11}(0,0)+b_{12}(0,0)+c_{1}(0) & =0 \\
b_{11}(0,0)+2 c_{1}(0) & =0 \\
b_{11}(0,0)+2 b_{12}(0,0) & =\frac{1}{a(0)} .
\end{aligned}
$$

By (4.7a) and (4.7b), we have

$$
\mathrm{b}_{11}(0,0)+2 \mathrm{~b}_{12}(0,0)=0 \text {. }
$$

We get a contradiction between (4.8) and the equality (4.7c). Hence codim $g \geqslant 4$ and so we complete the proof of (3).

Using the stated hypotheses we may verify statements (2) and (4) in a similar fashion.

Lemma 4.3. Let $g \in \varepsilon_{x, \lambda}\{x\}$ be a bifurcation problem.

(1) Let $\mathrm{g}=x\left(\mathrm{a} x+\mathrm{q} \lambda^{\mathrm{k}}\right)$, where $\mathrm{a}, \mathrm{q} \in \mathbb{R}$ and $\mathrm{aq} \neq 0, \mathrm{k} \geqslant 1$. If $\mathrm{p} \in\left\langle\mathrm{x}^{2}, x \lambda, \lambda^{\mathrm{k}+1}\right\rangle$, then

$$
\widetilde{\mathrm{T}}(\mathrm{g}+\mathrm{txp})=\widetilde{\mathrm{T}}(\mathrm{g}), \mathrm{t} \in[0,1] .
$$

(2) Let $\mathrm{g}=x\left(\mathrm{a} x^{\mathrm{l}}+\mathrm{q} \lambda\right)$, where $\mathrm{a}, \mathrm{q} \in \mathbb{R}$ and $\mathrm{aq} \neq 0, \mathrm{l} \geqslant 2$. If $\mathrm{p} \in\left\langle x^{\mathrm{l}+1}, x \lambda, \lambda^{2}\right\rangle$, then

$$
\widetilde{\mathrm{T}}(\mathrm{g}+\mathrm{txp})=\widetilde{\mathrm{T}}(\mathrm{g}), \mathrm{t} \in[0,1] .
$$

Proof. We only give the proof of (2), since the proof of (1) is similar.

Let $p=x^{l+1} b+x \lambda c+\lambda^{2} d$, where $b, c, d \in \varepsilon_{x, \lambda}$. Then

$$
g+t x p=x\left(a x^{l}+q \lambda+t x^{l+1} b+t x \lambda c+t \lambda^{2} d\right) .
$$


Let

$$
\Delta=a x^{l}+q \lambda+t x^{l+1} b+t x \lambda c+t \lambda^{2} d
$$

Then $g+\operatorname{txp}=x \Delta$ and

$$
\begin{aligned}
& \Delta_{x}=l a x^{l-1}+(l+1) t x^{l} b+t x^{l+1} b_{x}+t \lambda c+t x \lambda c_{x}+t \lambda^{2} d_{x} \\
& \Delta_{\lambda}=q+t x^{l+1} b_{\lambda}+t x c+t x \lambda c_{\lambda}+2 t \lambda d+t \lambda^{2} d_{\lambda} .
\end{aligned}
$$

Obviously, $\Delta, x \Delta_{x}, \lambda \Delta_{\lambda} \in<x^{l}, \lambda>$. Notice that $\widetilde{T}(g)=<x^{l}, \lambda>\{x\}$. Hence $\widetilde{T}(g+t x p) \subset \widetilde{T}(g)$ for all $\mathrm{t} \in[0,1]$.

On the other hand, since $\left.(q+t x c+t \lambda d)\right|_{(0,0)}=q \neq 0$, by (4.9),

$$
\lambda=\frac{1}{q+t x c+t \lambda d}\left[\Delta-(x+t x b) x^{l}\right] .
$$

Since

$$
x \Delta_{x}=l a x^{l}+(l+1) t x^{l+1} b+t x^{l+2} b_{x}+t x \lambda c+t x^{2} \lambda c_{x}+t x \lambda^{2} d_{x}
$$

and

$$
\begin{aligned}
{\left.\left[l a+(l+1) t x b+t x^{2} b_{x}\right]\right|_{(0,0)} } & =l a \neq 0, \\
x^{l} & =\frac{1}{l a+(l+1) t x b+t x^{2} b_{x}}\left[x \Delta_{x}-\left(t x c+t x^{2} c_{x}+t x \lambda d_{x}\right) \lambda\right] .
\end{aligned}
$$

By (4.10) and (4.11), we have

$$
x^{l}=\frac{1}{l a+(l+1) t x b+t x^{2} b_{x}}\left[x \Delta_{x}-\frac{t x c+t x^{2} c_{x}+t x \lambda d_{x}}{q+t x c+t \lambda d}\left(\Delta-(a+t x b) x^{l}\right)\right] .
$$

In (4.12), let

$$
L=\frac{1}{l a+(l+1) t x b+t x^{2} b_{x}}, \quad M=\frac{t x c+t x^{2} c_{x}+t x \lambda d_{x}}{q+t x c+t \lambda d}, \quad N=a+t x b
$$

Then

$$
x^{\mathrm{l}}=\mathrm{L}\left[\mathrm{x} \Delta_{\mathrm{x}}-\mathrm{M}\left(\Delta-\mathrm{N} x^{\mathrm{l}}\right)\right]=\mathrm{L} x \Delta_{x}-\mathrm{L} M \Delta+\mathrm{LMN} x^{\mathrm{l}} .
$$

Thus we have $(1-\mathrm{LMN}) \mathrm{x}^{\mathrm{l}}=\mathrm{L} x \Delta_{x}-\mathrm{LM} \Delta$.

Observe that $M(0,0)=0$ and $\left.(1-\operatorname{LMN})\right|_{(0,0)}=1 \neq 0$. Then

$$
\begin{aligned}
x^{l} & =\frac{1}{1-\operatorname{LMN}}\left(\mathrm{L} x \Delta_{x}-\mathrm{LM} \Delta\right) \in<\Delta, x \Delta_{x}>, \\
\lambda & \left.=\frac{1}{\mathrm{q}+\mathrm{t} x \mathrm{c}+\mathrm{t} \lambda \mathrm{d}}\left[\Delta-(\mathrm{a}+\mathrm{t} x \mathrm{~b}) x^{\mathrm{l}}\right)\right] \in<\Delta, x \Delta_{x}>.
\end{aligned}
$$

Hence $\widetilde{T}(g) \subset \widetilde{T}(g+t x p), t \in[0,1]$.

Lemma 4.4. Let $g \in \varepsilon_{x, \lambda}\{x\}$ be singular at $(0,0)$. If $g$ has the form $g(x, \lambda)=x\left(x^{l} a+x \lambda b+\lambda^{k} q\right)$, where $a \in \varepsilon_{x}, b, q \in \varepsilon_{x, \lambda}$ and $a(0) \neq 0, q(0,0) \neq 0$, then

$$
g(x, \lambda) \sim \widetilde{g}(x, \lambda)=x\left[x^{l} a(0)+\lambda^{k} q(0,0)\right],
$$

where $l=1, k \in \mathbb{N}$ or $k=1, l \in \mathbb{N}$.

Proof. If $l=1, k \in \mathbb{N}$, then $\widetilde{g}(x, \lambda)=x\left[a(0) x+q(0,0) \lambda^{k}\right]$ and

$$
g(x, \lambda)=x\left[x a(x)+x \lambda b(x, \lambda)+\lambda^{k} q(x, \lambda)\right]=x\left[a(0) x+q(0,0) \lambda^{k}+p\right]=\widetilde{g}(x, \lambda)+x p,
$$

where $p \in\left\langle x^{2}, x \lambda, \lambda^{k+1}>\right.$. By Theorem 3.3 and Lemma 4.3, we have 


$$
\widetilde{g}(x, \lambda) \sim \widetilde{g}(x, \lambda)+\operatorname{txp}(x, \lambda), t \in[0,1] .
$$

Hence $g(x, \lambda) \sim \widetilde{g}(x, \lambda)$. If $k=1, l \in \mathbb{N}$, then $\widetilde{g}(x, \lambda)=x\left[a(0) x^{l}+q(0,0) \lambda\right]$ and

$$
g(x, \lambda)=x\left[x^{l} a(x)+x \lambda b(x, \lambda)+\lambda q(x, \lambda)\right]=x\left[a(0) x^{l}+q(0,0) \lambda+p\right]=\widetilde{g}(x, \lambda)+x p,
$$

where $p \in\left\langle x^{l+1}, x \lambda, \lambda^{2}>\right.$. By Theorem 3.3 and Lemma 4.3, we have

$$
\widetilde{g}(x, \lambda) \sim \widetilde{g}(x, \lambda)+\operatorname{txp}(x, \lambda), t \in[0,1] .
$$

Hence $g(x, \lambda) \sim \widetilde{g}(x, \lambda)$.

Theorem 4.5. Let $g \in \varepsilon_{x, \lambda}\{x\}$ satisfy $g=g_{x}=0$ at $(0,0)$ with codim $g \leqslant 3$. Then

(1) g must be $t$-equivalent to one of the bifurcation problems: $\varepsilon x^{k}+\delta \lambda^{l} x$, where $(k, l)$ may equal to $(2,1),(3,1)$, $(4,1),(2,2),(2,3)$ and $\varepsilon, \delta= \pm 1$;

(2) any two cases in (1) are not equivalent to each other.

Proof.

(1) Let $g \in \varepsilon_{x, \lambda}\{x\}$ be a bifurcation problem and codim $g \leqslant 3$. By Lemma 4.1, we set

$$
g(x, \lambda)=x\left(x^{n} a(x)+\lambda q(x, \lambda)\right),
$$

where $n \in \mathbb{N}, a \in \varepsilon_{x}, q \in \varepsilon_{x, \lambda}$ and $a(0) \neq 0$. By Lemma 4.2, we conclude $n \leqslant 3$. Let

$$
f(x, \lambda)=x^{n} a(x)+\lambda q(x, \lambda) .
$$

Then we will discuss with different values of $n$ as below.

(i) Let $n=3$. Then

$$
g(x, \lambda)=x\left(x^{3} a(x)+\lambda q(x, \lambda)\right), f(x, \lambda)=x^{3} a(x)+\lambda q(x, \lambda) .
$$

If $\mathrm{f}_{\lambda}(0,0) \neq 0$, then $\mathrm{q}(0,0) \neq 0$. By Lemma 4.4 ,

$$
g(x, \lambda) \sim \tilde{g}(x, \lambda)=x\left[a(0) x^{3}+q(0,0) \lambda\right] .
$$

Let

Then $(S,(X, \Lambda)) \in \Gamma$ and

$$
S(x, \lambda)=\sqrt[3]{|\mathbf{a}(0)|}, \quad x(x, \lambda)=\frac{1}{\sqrt[3]{|\mathbf{a}(0)|}} x, \quad \Lambda(\lambda)=\frac{1}{|\mathbf{q}(0,0)|} \lambda
$$

$$
S(x, \lambda) \tilde{g}(X(x, \lambda), \wedge(\lambda))=\varepsilon x^{4}+\delta x \lambda,
$$

where $\varepsilon=\operatorname{sgn}(a(0)), \delta=\operatorname{sgn}(q(0,0))$. Hence $g(x, \lambda) \sim \varepsilon x^{4}+\delta x \lambda$, which is the case of $(k, l)=(4,1)$.

If $f_{\lambda}(0,0)=0$, by Lemma 4.2 , we have codim $g \geqslant 4$.

(ii) Let $\mathrm{n}=2$. Then

$$
g(x, \lambda)=x\left(x^{2} a(x)+\lambda q(x, \lambda)\right), f(x, \lambda)=x^{2} a(x)+\lambda q(x, \lambda) .
$$

If $\mathrm{f}_{\lambda}(0,0) \neq 0$, then $\mathrm{q}(0,0) \neq 0$. By Lemma 4.4 ,

$$
g(x, \lambda) \sim \tilde{g}(x, \lambda)=x\left(a(0) x^{2}+q(0,0) \lambda\right) .
$$

Let

$$
S(x, \lambda)=\sqrt{|\mathbf{a}(0)|}, \quad X(x, \lambda)=\frac{1}{\sqrt{|\mathbf{a}(0)|}} x, \quad \Lambda(\lambda)=\frac{1}{|\mathbf{q}(0,0)|} \lambda .
$$

Then $(S,(X, \Lambda)) \in \Gamma$ and

$$
S(x, \lambda) \tilde{g}(X(x, \lambda), \Lambda(\lambda))=\varepsilon x^{3}+\delta x \lambda,
$$

where $\varepsilon=\operatorname{sgn}(a(0)), \delta=\operatorname{sgn}(q(0,0))$. Hence $g(x, \lambda) \sim \varepsilon x^{3}+\delta x \lambda$, which is the case of $(k, l)=(3,1)$.

If $f_{\lambda}(0,0)=0$, by Lemma 4.2 , we have codim $g \geqslant 4$. 
(iii) Let $n=1$. Then

$$
g(x, \lambda)=x[x a(x)+\lambda q(x, \lambda)], f(x, \lambda)=x a(x)+\lambda q(x, \lambda) .
$$

If $\mathrm{f}_{\lambda}(0,0) \neq 0$, then $\mathrm{q}(0,0) \neq 0$. By Lemma 4.4 ,

$$
g(x, \lambda) \sim \tilde{g}(x, \lambda)=x[a(0) x+q(0,0) \lambda] .
$$

Let

Then we conclude that

$$
S(x, \lambda)=|a(0)|, \quad X(x, \lambda)=\frac{1}{|a(0)|} x, \quad \Lambda(\lambda)=\frac{1}{|q(0,0)|} \lambda .
$$

$$
g(x, \lambda) \sim \varepsilon x^{2}+\delta x \lambda,
$$

where $\varepsilon=\operatorname{sgn}(a(0)), \delta=\operatorname{sgn}(q(0,0))$. This is the case of $(k, l)=(2,1)$.

If $f_{\lambda}(0,0)=0$, then there exist $q_{1}, q_{2} \in \varepsilon_{x, \lambda}$ such that $q=x q_{1}+\lambda q_{2}$ and hence

$$
g(x, \lambda)=x\left(x a+x \lambda q_{1}+\lambda^{2} q_{2}\right), f(x, \lambda)=x a+x \lambda q_{1}+\lambda^{2} q_{2} .
$$

Here we consider two cases:

If $\mathrm{f}_{\lambda \lambda}(0,0) \neq 0$, then $\mathrm{q}_{2}(0,0) \neq 0$. By Lemma 4.4 ,

$$
g(x, \lambda) \sim \tilde{g}(x, \lambda)=x\left[a(0) x+q_{2}(0,0) \lambda^{2}\right] .
$$

Let

Then we conclude that

$$
S(x, \lambda)=|a(0)|, \quad X(x, \lambda)=\frac{1}{|a(0)|} x, \quad \Lambda(\lambda)=\frac{1}{\left|q_{2}(0,0)\right|} \lambda .
$$

$$
g(x, \lambda) \sim \varepsilon x^{2}+\delta x \lambda^{2}
$$

where $\varepsilon=\operatorname{sgn}(a(0)), \delta=\operatorname{sgn}\left(q_{2}(0,0)\right)$. This is the case of $(k, l)=(2,2)$.

If $f_{\lambda \lambda}(0,0)=0$, then $q_{2}(0,0)=0$. There exist $q_{3}, q_{4} \in \varepsilon_{x, \lambda}$ such that $q_{2}=x q_{3}+\lambda q_{4}$. Hence

$$
g=x\left(x a+x \lambda q_{1}+x \lambda^{2} q_{3}+\lambda^{3} q_{4}\right), f=x a+x \lambda q_{1}+x \lambda^{2} q_{3}+\lambda^{3} q_{4} .
$$

If $f_{\lambda \lambda \lambda}(0,0)=0$, by Lemma 4.2 , we have codim $g \geqslant 4$.

If $f_{\lambda \lambda \lambda}(0,0) \neq 0$, then $q_{4}(0,0) \neq 0$. By Lemma 4.4 ,

$$
g(x, \lambda) \sim \tilde{g}(x, \lambda)=x\left(a(0) x+q_{4}(0,0) \lambda^{3}\right) .
$$

Let

Then we have

$$
S(x, \lambda)=|a(0)|, \quad X(x, \lambda)=\frac{1}{|a(0)|} x, \quad \Lambda(\lambda)=\frac{1}{\sqrt[3]{\left|q_{4}(0,0)\right|}} \lambda
$$

$$
g(x, \lambda) \sim \varepsilon x^{2}+\delta x \lambda^{3}
$$

where $\varepsilon=\operatorname{sgn}(a(0)), \delta=\operatorname{sgn}\left(q_{4}(0,0)\right)$. This is the case of $(k, l)=(2,3)$.

(2) The proof contains three steps.

(i) Obviously, the germs with different codimensions cannot be equivalent to each other.

(ii) We observe that bifurcation problems with codimension $2, \chi\left(\varepsilon \chi^{2}+\delta \lambda\right)$ and $\chi\left(\varepsilon x+\delta \lambda^{2}\right)$, are not equivalent. We use symbol $N(g, \lambda)$ to represent the number of $x$ that satisfies the equation $g(x, \lambda)=0$. If $g \sim h$, then it is obvious that $N(h, \lambda)=N(g, \Lambda(\lambda)), \lambda \in(\mathbb{R}, 0)$. Assume the abscissa is $\lambda$-axe and the ordinate is $x$-axe. We only discuss $\varepsilon=1, \delta=-1$, since the other cases are similar.

On the left of $(0,0)$,

$$
\mathrm{N}\left[x\left(x^{2}-\lambda\right), \lambda\right]=1, \mathrm{~N}\left[x\left(x-\lambda^{2}\right), \lambda\right]=2
$$

On the right of $(0,0)$,

$$
N\left[x\left(x^{2}-\lambda\right), \lambda\right]=3, N\left[x\left(x-\lambda^{2}\right), \lambda\right]=2 .
$$

Hence $x\left(x^{2}-\lambda\right)$ and $x\left(x-\lambda^{2}\right)$ are not equivalent. 
(iii) We consider bifurcation problems with codimension 3, that is, $x\left(\varepsilon x^{3}+\delta \lambda\right), x\left(\varepsilon x+\delta \lambda^{3}\right)$. We only disscuss $\varepsilon=\delta=1$. Suppose

$$
x\left(x^{3}+\lambda\right) \sim x\left(x+\lambda^{3}\right) .
$$

Then there exists $(S,(X, \Lambda)) \in \Gamma$ such that

$$
S(x, \lambda)\left(X^{4}(x, \lambda)+X(x, \lambda) \Lambda(\lambda)\right)=x^{2}+x \lambda^{3} .
$$

Set $\lambda=0$. Then $S(x, 0) X^{4}(x, 0)=x^{2}$, that is,

$$
\sqrt[2]{S(x, 0)} X^{2}(x, 0)=|x| .
$$

In (4.13), the function on the left is differentiable about $x$, but the function on the right is not. This is a contradiction and so the assumption is not true. It is similar to prove other cases.

\section{Acknowledgment}

This work is partially supported by NSFC grant No. 11671070, No. 11501051.

\section{References}

[1] O. Diekmann, A beginner's guide to adaptive dynamics, Mathematical modelling of population dynamics, Banach Center Publ., Polish Acad. Sci. Inst. Math., Warsaw, 63 (2003), 47-86. 1

[2] U. Dieckmann, R. Law, The dynamical theory of coevolution: a derivation from stochastic ecological processes, J. Math. Biol., 34 (1997), 569-612. 1

[3] S.-P. Gao, Y.-C. Li, Classification of $\left(\mathrm{D}_{4}, \mathrm{~S}^{1}\right)$-equivariant bifurcation problems up to topological codimension 2, Sci. China Ser. A, 46 (2003), 862-871. 1

[4] S. Geritz, J. Metz, É. Kisdi, G. Meszéna, Dynamics of adaptation and evolutionary branching, Phys. Rev. Lett., 78 (1997), 2024-2027. 1

[5] M. Golubitsky, M. Roberts, A classification of degenerate Hopf bifurcations with $\mathrm{O}(2)$ symmetry, J. Differential Equations, 69 (1987), 216-264. 1

[6] M. Golubitsky, D. G. Schaeffer, A theory for imperfect bifurcation via singularity theory, Comm. Pure Appl. Math., 32 (1979), 21-98. 1

[7] M. Golubitsky, D. G. Schaeffer, Singularities and groups in bifurcation theory, Vol. I, Applied Mathematical Sciences, Springer-Verlag, New York, (1985). 1

[8] B. L. Keyfitz, Classification of one-state-variable bifurcation problems up to codimension seven, Dynam. Stability Systems, 1 (1986), 1-41. 1

[9] M. Manoel, I. Stewart, The classification of bifurcations with hidden symmetries, Proc. London Math. Soc., 80 (2000), 198-234. 1

[10] J. Martinet, Singularities of smooth functions and maps, Translated from the French by Carl P. Simon, London Mathematical Society Lecture Note Series, Cambridge University Press, Cambridge-New York, (1982). 1

[11] M. Peters, Classification of two-parameter bifurcations, Singularity theory and its applications, Part II, Coventry, (1988/1989), Lecture Notes in Math., Springer, Berlin, 1463 (1991), 294-300. 1

[12] J. M. Smith, G. R. Price, The logic of animal conflict, Nature, 246 (1973), 15-18. 1

[13] A. Vutha, M. Golubitsky, Normal forms and unfoldings of singular strategy functions, Dyn. Games Appl., 5 (2015), 180-213. 1

[14] X.-H. Wang, M. Golubitsky, Singularity theory of fitness functions under dimorphism equivalence, J. Math. Biol., 73 (2016), 526-573. 1

[15] D. Waxman, S. Gavrilets, 20 questions on adaptive dynamics, J. Evol. Biol., 18 (2005), 1139-1154. 1 